07.2;07.3

\title{
Исследование шумовых характеристик вертикально-излучающих лазеров с ромбовидной токовой апертурой для применения в компактном квантовом цезиевом магнитометре
}

\author{
(C) М.А. Бобров ${ }^{1}$, С.А. Блохин ${ }^{1}$, Н.А. Малеев ${ }^{1}$, А.Г. Кузьменков ${ }^{2}$, А.А. Блохин ${ }^{1}$, А.П. Васильев ${ }^{2}$, \\ М.М. Кулагина ${ }^{1}$, А.С. Пазгалев ${ }^{1}$, И.И. Новиков ${ }^{3}$, Л.Я. Карачинский ${ }^{4}$, В.М. Устинов ${ }^{2}$ \\ ${ }^{1}$ Физико-технический институт им. А.Ф. Иофффе РАН, Санкт-Петербург, Россия \\ ${ }^{2}$ Научно-технологический центр микроэлектроники и субмикронных гетероструктур РАН, Санкт-Петербург, Россия \\ ${ }^{3}$ Университет ИТМО, Санкт-Петербург, Россия \\ ${ }^{4}$ ООО „Коннектор Оптикс“, Санкт-Петербург, Россия \\ E-mail: bobrov.mikh@gmail.com
}

Поступило в Редакцию 30 июня 2021 г.

В окончательной редакции 5 августа 2021 г.

Принято к публикации 25 августа 2021 г.

\begin{abstract}
Исследована возможность использования вертикально-излучающих лазеров диапазона $895 \mathrm{~nm}$ с внутрирезонаторными контактами (ВК-ВИЛ) и ромбовидной оксидной токовой апертурой для создания компактного квантового магнитометра с оптической накачкой газовой ячейки с атомами ${ }^{133} \mathrm{Cs}$, работающего в ненулевых магнитных полях, для применения в магнитоэнцефалографических системах. Проведен анализ амплитудных и поляризационных шумов ВК-ВИЛ с различными потерями на вывод излучения и различной шириной спектральной линии в частотном диапазоне от $1 \mathrm{~Hz}$ до $100 \mathrm{kHz}$. Показано, что ВК-ВИЛ с низкими потерями на вывод излучения (малой шириной спектральной линии) обладают поляризационными шумами, сравнимыми с амплитудными шумами. При выходной оптической мощности $0.8 \mathrm{~mW}$ и ширине спектральной линии $55 \mathrm{MHz}$ уровень шумов, измеренный в полосе $1 \mathrm{~Hz}$, составляет $148 \mathrm{~dB} / \mathrm{Hz}$ при частоте $40 \mathrm{kHz}$. Предельная вариационная чувствительность квантового магнитометра с оптической накачкой с рабочей частотой $40 \mathrm{kHz}$ для исследованных ВК-ВИЛ оценена на уровне $16 \mathrm{fT} / \sqrt{\mathrm{Hz}}$ для однолучевой $M_{Z}$-схемы и порядка $\sim 11 \mathrm{fT} / \sqrt{\mathrm{Hz}}$ для двулучевой $M_{X}$-схемы.
\end{abstract}

Ключевые слова: вертикально-излучающий лазер, квантовый магнитометр с оптической накачкой, амплитудные шумы, поляризационные шумы.

DOI: 10.21883/PJTF.2021.24.51789.18940

В настоящее время для регистрации сверхмалых магнитных полей, создаваемых электрической активностью головного мозга, все шире используется быстро развивающаяся неинвазивная технология магнитоэнцефалографии (МЭГ) [1]. Характерные величины сверхмалых магнитных полей, создаваемых головным мозгом, не превышают нескольких сотен fT. В традиционных МЭГ-системах, позволяющих регистрировать такие сверхмалые магнитные поля с чувствительностью $\sim 3 \mathrm{fT} / \sqrt{\mathrm{Hz}}$, используется массив магнитных датчиков на основе сверхпроводящих квантовых интерференционных устройств (англ. superconducting quantum interference device) [2]. К существенным недостаткам таких МЭГ-систем можно отнести необходимость создания магнитоэкранированной комнаты (единицы nT) и обеспечения постоянного охлаждения магнитных датчиков до температур жидкого гелия $\left(-270^{\circ} \mathrm{C}\right)$, что приводит к существенному росту себестоимости и размеров самой системы. Использование шлема Дьюара фиксированной формы с массивом датчиков, рассчитанного на максимальный размер головы пациента, существенно усложняет проведение исследований мозга у подвижных пациентов, что приводит к падению пространственного разрешения.
Недавно был продемонстрирован альтернативный вариант МЭГ-системы с оптической накачкой, не требующий охлаждения и неподвижного положения пациента, в котором используются коммерчески доступные квантовые магнитометры с оптической накачкой (КМOH, англ. optically pumped atomic magnetometer) [3], работающие в режиме, свободном от спин-обменного уширения (англ. spin exchange relaxation-free, SERF) и обладающие чувствительностью $\sim 10 \mathrm{fT} / \sqrt{\mathrm{Hz}}$ в полосе частот $1-130 \mathrm{~Hz}$ [4]. Принцип действия таких магнитометров основан на оптически детектируемом магнитном резонансе в атомах щелочного металла, находящегося в газовой фазе. Существенными достоинствами КМОН с использованием схемы SERF являются высокая предельная чувствительность и малые размеры самого датчика, что позволяет увеличить регистрируемый сигнал и улучшить пространственное разрешение. В то же время недостатками такого датчика являются необходимость обеспечения сверхслабого однородного магнитного поля (единицы nT) и сильное взаимное влияние расположенных рядом датчиков (низкочастотный диапазон работы).

В недавней работе [5] была показана возможность получения сравнимой с достигаемой в КМОН в режиме SERF чувствительности для оптически накачи- 
ваемого магнитометра, реализованного в двулучевой $M_{X}$-схеме [6]. В данном магнитометре использовался эффект сужения линии магнитного резонанса в условиях высокой мощности оптической накачки и больших концентраций щелочных атомов при ненулевых магнитных полях (единицы $\mu \mathrm{T}$ ) [7]. К преимуществам МЭГ-системы на базе такого датчика относятся существенное снижение требований к экранированию комнаты для проведения исследований (снижение габаритов и себестоимости системы) и уход рабочей частоты из низкочастотной области (КМOH в режиме SERF) в килогерцевый диапазон (уменьшение влияния низкочастотных шумов окружающего оборудования и лазерного источника). Ключевыми компонентами таких устройств являются инжекционный одномодовый лазерный источник и миниатюрная газовая ячейка со щелочным металлом (например, $\mathrm{K}, \mathrm{Rb}$ или

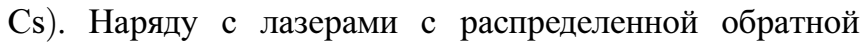
связью (РОС-лазеры, англ. DFB-laser) для реализации КМОН могут быть использованы вертикально-излучающие лазеры (ВИЛ, англ. vertical-cavity surface-emitting lasers, VCSEL) [8], которые потенциально позволяют улучшить компактность МЭГ-систем. Другими потенциально важными приложениями ВИЛ с длиной волны излучения, настроенной на один из резонансов щелочного металла, являются компактные гироскопы на основе эффекта ядерного магнитного резонанса и компактные атомные стандарты частоты на основе эффекта когерентного пленения населенности [8]. Рассматриваемые лазерные излучатели должны обладать возможностью прецизионной настройки длины волны на используемую спектральную линию щелочного металла, пространственноодномодовым излучением с малой спектральной шириной линии излучения (менее $100 \mathrm{MHz}$ ), фиксированным направлением линейной поляризации и низким уровнем шумов лазерного излучения. При этом любая нестабильность лазерного излучения, например амплитудный шум (англ. relative intensity noise, RIN) лазера, скачкообразное изменение поляризации (англ. polarization-hop) и/или перескок мод в спектре (англ. frequency mode-hop) при изменении тока накачки напрямую сказываются на метрологических характеристиках КМОН (на отношении сигнал/шум/ширина магнитного резонанса). В связи с этим детальное исследование шумовых характеристик ВИЛ представляется крайне важным для дальнейшего улучшения характеристик КМОН $[9,10]$.

В настоящей работе экспериментально исследована интенсивность амплитудных и поляризационных шумов разработанных ВИЛ спектрального диапазона $895 \mathrm{~nm}$. Рассмотрено влияние спектральной ширины линии излучения, зависящей от потерь на вывод излучения лазера, на шумовые характеристики ВИЛ.

Исследуемые одномодовые ВИЛ спектрального диапазона $895 \mathrm{~nm}$ реализованы на основе ранее предложенной конструкции ВИЛ с внутрирезонаторными контактами и ромбовидной оксидной токовой апертурой (ВК-ВИЛ), ее основные особенности и процесс изготовления лазеров представлены в работах $[11,12]$. Для исследования влияния уровня потерь на вывод излучения на шумовые характеристики ВИЛ были изготовлены приборы с различным количеством пар в верхнем диэлектрическом распределенном брэгговском отражателе. Ватт-амперные характеристики ВК-ВИЛ с различными потерями на вывод излучения $A_{m}$, измеренные в непрерывном режиме при $20^{\circ} \mathrm{C}$, приведены на рис. 1 . Значения $A_{m}$ получены в рамках метода матриц переноса в приближении отсутствия внутренних потерь в зеркалах. ВК-ВИЛ с высокими потерями на вывод излучения $-A_{m} \sim 0.71 \%$ на полный проход (туда-обратно) лазерного резонатора (далее ВК-ВИЛ-1, на рисунках IC-VCSEL-1) - обладает пороговым током $\sim 0.8 \mathrm{~mA}$, дифференциальной эффективностью $\sim 0.65 \mathrm{~W} / \mathrm{A}$ и выходной оптической мощностью $\sim 1.4 \mathrm{~mW}$ при токе накачки $3 \mathrm{~mA}$. Тогда как ВК-ВИЛ с более низким уровнем потерь на вывод излучения $-A_{m} \sim 0.23 \%$ (далее ВК-ВИЛ-2, на рисунках IC-VCSEL-2) - обладает пороговым током $\sim 0.5 \mathrm{~mA}$, дифференциальной эффективностью $\sim 0.37 \mathrm{~W} / \mathrm{A}$ и выходной оптической мощностью $0.9 \mathrm{~mW}$ при токе накачки $3 \mathrm{~mA}$. Оценка уровня внутренних потерь, полученная из зависимости эффективности вывода излучения от уровня потерь на вывод излучения $T_{m}$, для двух исследуемых лазеров дает значение $\sim 0.35 \pm 0.05 \%$. Относительно высокий уровень внутренних потерь обусловлен ростом оптических потерь из-за рассеяния и/или дифракции света на оксидной токовой апертуре при уменьшении размеров апертуры [10]. Согласно данным анализа спектров лазерного излучения, уровень подавления мод высшего порядка по отношению к фундаментальной моде в рассматриваемом диапазоне токов для обоих лазеров превышает $30 \mathrm{~dB}$. Поляризационные характеристики исследуемых приборов демонстрируют доминирование одного направления поляризации излучения с фактором подавления ортогональной поляризации более $20 \mathrm{~dB}$ при токах накачки более $2 \mathrm{~mA}$. Также были проведены измерения спектральной ширины лазерного излучения в зависимости от тока инжекции лазера при температуре $20^{\circ} \mathrm{C}$ с помощью сканирующего интерферометра Фабри-Перо Thorlabs SA-200 (с разрешением 7.5 MHz). Увеличение потерь на вывод излучения ведет к уширению линии излучения. Так, при токе инжекции более $2 \mathrm{~mA}$ для обоих лазеров ширина спектральной линии для ВК-ВИЛ-1 составила 120-160 MНz, а для ВК-ВИЛ-2 60-70 MHz (рис. 1). Следует отметить, что неклассическое поведение ширины линии излучения для ВК-ВИЛ-2 при выходной оптической мощности более $0.3 \mathrm{~mW}$, повидимому, обусловлено падением дифференциального усиления и, как следствие, ростом $\alpha$-фактора в условиях высокой плотности носителей и фотонов в микрорезонаторе [13].

Существенное влияние на предельную вариационную чувствительность атомного магнитометра оказывает уровень амплитудных и/или поляризационных шумов (англ. polarization-resolved RIN) лазера. При этом КМОН, реализованные по классической однолучевой 

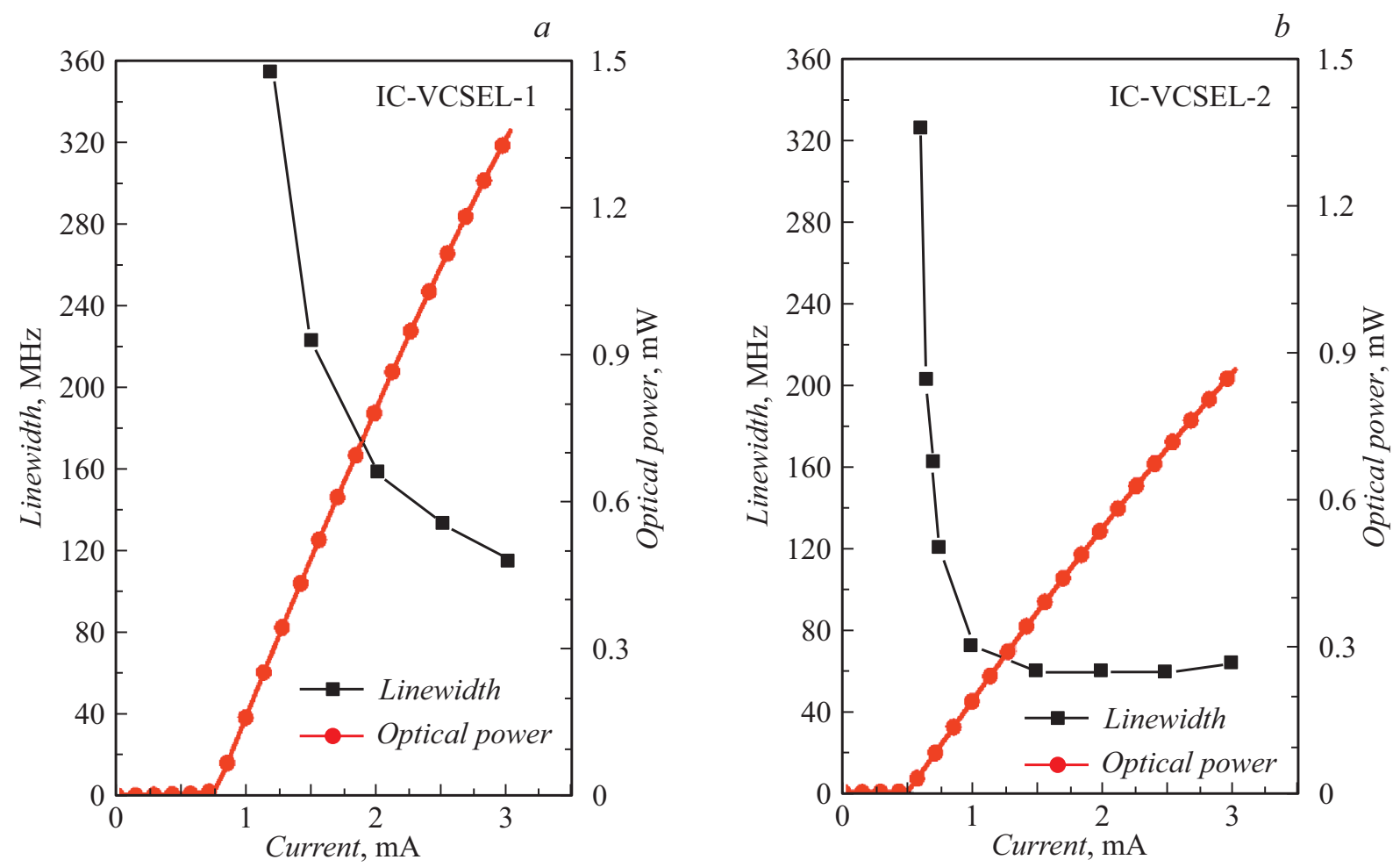

Рис. 1. Ватт-амперные характеристики и спектральная ширина линии излучения для ВК-ВИЛ-1 (IC-VCSEL-1) (a) и ВК-BИЛ-2 $($ IC-VCSEL-2) $(b)$ с характерным размером ромбовидной токовой оксидной апертуры $\sim 2.5 \mu \mathrm{m}$ в зависимости от тока накачки. Измерения проведены при температуре $20^{\circ} \mathrm{C}$.

$M_{Z}$-схеме, менее чувствительны к нестабильности поляризации лазерного излучения в отличие от атомных магнитометров, реализованных на базе двулучевых $M_{X}$-схем. Измерение амплитудных шумов ВК-ВИЛ осуществлялось с помощью малошумящего фотодетектора Hamamatsu S3584 и синхронного детектора Lock-in SR830 в режиме измерения плотности шумов в полосе $1 \mathrm{~Hz}$. На рис. 2, a представлены экспериментальные зависимости уровня амплитудных шумов от выходной оптической мощности лазеров, измеренные при температуре $20^{\circ} \mathrm{C}$ и частоте $40 \mathrm{kHz}$ (обусловлено схемой измерения цезиевого магнитного резонанса [5]). Для обоих типов лазеров при превышении порога генерации с ростом выходной оптической мощности наблюдается практически одинаковое падение амплитудных шумов с их последующим насыщением на уровне $-148 \mathrm{~dB} / \mathrm{Hz}$ при оптической мощности более $0.5 \mathrm{~mW}$.

Измерения поляризационных шумов лазеров проводились при температуре $20^{\circ} \mathrm{C}$ и частоте $40 \mathrm{kHz}$ с использованием балансной схемы детектирования. Излучение исследуемого лазера направлялось на поляризационный светоделительный кубик и собиралось двумя фотодетекторами, подключенными к балансной схеме детектирования. Лазерное излучение попадало на поляризационный светоделительный кубик под углом $45^{\circ}$ для возможности регистрации угла наклона плоскости поляризации, т. е. измерения второго параметра Стокса. Далее разностный сигнал направлялся на синхронный детектор и в режиме измерения плотности шумов в полосе $1 \mathrm{~Hz}$ регистрировалась интенсивность поляризационных шумов лазера, состоящих из шумов двух ортогональных мод. Как показано на рис. 2, $b$, уровень поляризационных шумов для ВК-ВИЛ-1, измеренный при выходной оптической мощности $0.8 \mathrm{~mW}$, превышает ожидаемый расчетный уровень дробового шума детектируемого излучения на $18 \mathrm{~dB} / \mathrm{Hz}$. В то же время для ВК-ВИЛ-2 эта разница составляет $4 \mathrm{~dB} / \mathrm{Hz}$, а уровень поляризационных шумов снижается до уровня амплитудных шумов. Существенное увеличение поляризационных шумов ВК-ВИЛ-1 по сравнению с таковыми в случае ВК-ВИЛ-2, по-видимому, связано с большей спектральной шириной линии лазерного излучения и, как следствие, увеличением дрожания плоскости поляризации выходного излучения лазера. Поиск причин такого поведения требует более детальных исследований, что выходит за рамки настоящей работы.

Для определения частотно-шумовых характеристик исследуемых лазеров были проведены измерения амплитудных и поляризационных шумов в зависимости от частоты в диапазоне от $1 \mathrm{~Hz}$ до $100 \mathrm{kHz}$. Согласно рис. 3, для лазера ВК-ВИЛ-2 зависимости для поляризационных и амплитудных шумов от частоты практически совпадают и близки к кривой для амплитудных шумов лазера ВК-ВИЛ-1. Амплитудные шумы практически одинаковы для обоих исследованных лазеров, их уровень в области частот $40 \mathrm{kHz}$ ниже $-120 \mathrm{~dB} / \mathrm{Hz}$. Следует отметить, что 

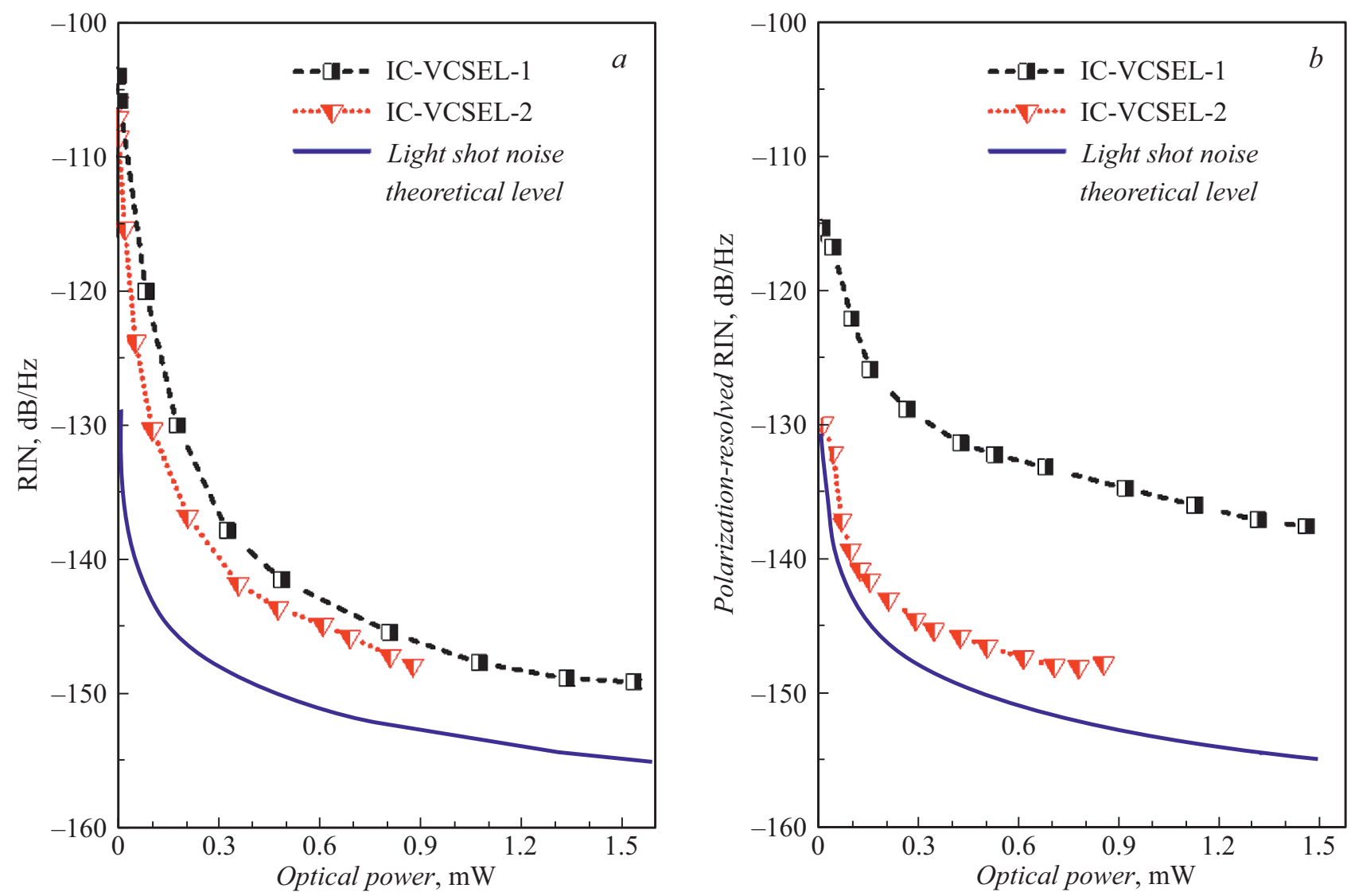

Pис. 2. Зависимости амплитудных (RIN) (a) и поляризационных (polarization-resolved RIN) (b) шумов от выходной оптической мощности для двух типов ВК-ВИЛ с различными потерями на вывод излучения, измеренные в полосе $1 \mathrm{~Hz}$ на частоте $40 \mathrm{kHz}$. Измерения проведены при температуре $20^{\circ} \mathrm{C}$. Сплошная кривая - расчетный уровень дробового шума детектируемого излучения.

величина амплитудных шумов ВК-ВИЛ-2 в области частот $10 \mathrm{kHz}$ сравнима с результатами, полученными для ВИЛ спектрального диапазона $895 \mathrm{~nm}$ с расширенным резонатором и субволновой решеткой на поверхности верхнего распределенного брэгговского отражателя (для фиксации поляризации), имеющего ширину линии $23 \mathrm{MHz}$ и оптическую мощность $0.15 \mathrm{~mW}$ [14]. В то же время частотно-зависимые поляризационные шумы ВК-ВИЛ-1 на $\sim 13 \mathrm{~dB} / \mathrm{Hz}$ выше, чем поляризационные шумы ВК-ВИЛ-2, что следует учитывать при выборе схемы реализации КМОН.

Оценка предельной вариационной чувствительности КМОН осуществлялась по критерию отношения крутизны сигнала, т. е. отношения амплитуды сигнала к ширине резонансной линии, к спектральной плотности шума детектирующего излучения [15]. Для оценки достижимой вариационной чувствительности атомного магнитометра ненулевого поля, реализуемого на базе исследуемых ВК-ВИЛ, были использованы измеренные параметры компактной кубической $\left(0.125 \mathrm{~cm}^{3}\right)$ газовой ячейкой $\mathrm{c}$ парами ${ }^{133} \mathrm{Cs}$ и азота под давлением 100 Torr, нагретой до $85^{\circ} \mathrm{C}$, имеющей ширину линии магнитного резонанса $1 \mathrm{kHz}$ [16]. Согласно оценкам, атомный магнитометр на базе однолучевой $M_{Z}$-схемы с рабочей частотой $40 \mathrm{kHz}$ при использовании любого исследуемого ВК-ВИЛ потенциально может иметь предельную вариационную чувствительность около $16 \mathrm{fT} / \sqrt{\mathrm{Hz}}$. В случае использования двулучевой $M_{X}$-схемы предельная вариационная чувствительность для ВК-ВИЛ-1 достигает $57 \mathrm{fT} / \sqrt{\mathrm{Hz}}$, тогда как для ВК-ВИЛ-2 чувствительность может быть улучшена до уровня $11 \mathrm{fT} / \sqrt{\mathrm{Hz}}$. Для сравнения измеренная чувствительность компактного магнитометра в $M_{X}$-схеме с использованием цезиевой ячейки ВИЛ с мощностью $0.15 \mathrm{~mW}$ в канале детектирования и ВИЛ с мощностью $0.35 \mathrm{~mW}$ в канале накачки составила $300 \mathrm{fT} / \sqrt{\mathrm{Hz}}$ в скалярном режиме и $16 \mathrm{fT} / \mathrm{cm} / \sqrt{\mathrm{Hz}}$ в градиентометрической схеме [17]. Полученные результаты подтверждают потенциальную возможность применения разработанных ВК-ВИЛ при создании магнитных сенсоров для МЭГ-систем, реализованных на основе КМОН

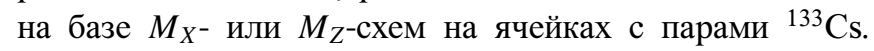
Следует отметить, что низкий уровень поляризационных шумов ВК-ВИЛ позволяет использовать их внутри компактных КМОН без дополнительной фильтрации поляризационных шумов в канале детектирования, например, с помощью поляризатора или поляризационного светоделительного кубика (англ. polarizing beamsplitter cube). 

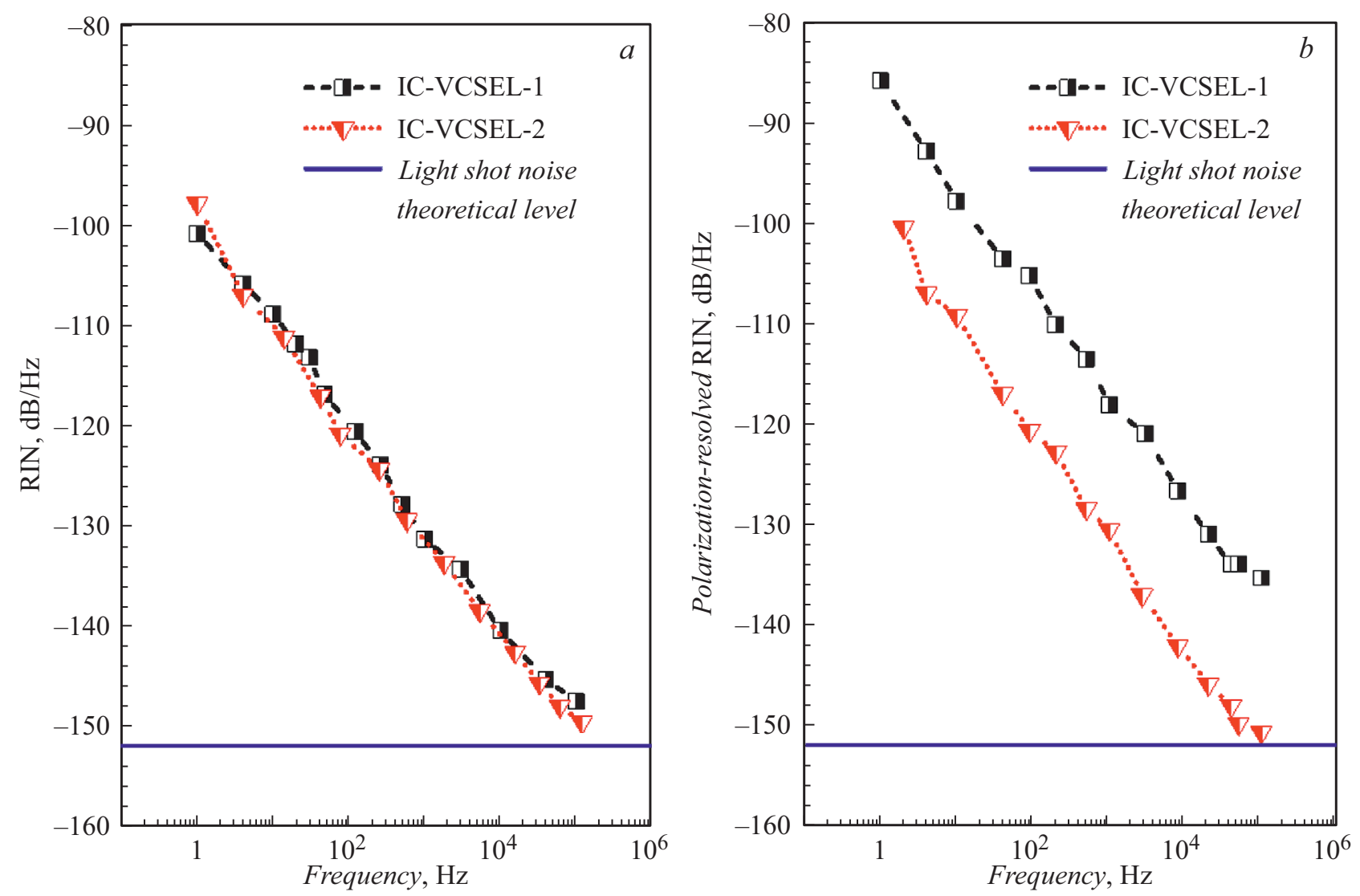

Рис. 3. Частотные зависимости амплитудных (RIN) (a) и поляризационных (polarization-resolved RIN) (b) шумов для двух типов ВК-ВИЛ с различными потерями на вывод излучения, измеренные в полосе $1 \mathrm{~Hz}$ при оптической мощности излучения лазера $0.8 \mathrm{~mW}$. Измерения проведены при температуре $20^{\circ} \mathrm{C}$. Сплошная кривая - расчетный уровень дробового шума детектируемого излучения.

В работе были проведены исследования амплитудных и поляризационных шумов вертикально-излучающих лазеров с внутрирезонаторными контактами и ромбовидной токовой апертурой с различными потерями на вывод излучения. Обнаружено, что для ВК-ВИЛ с низкими потерями на вывод излучения и шириной спектральной линии излучения 50-60 MHz при оптической мощности 0.5-1.0 mW поляризационные шумы становятся сравнимыми с амплитудными шумами, что делает возможным применение данных лазеров в различных оптических схемах КМОН. Применение таких ВК-ВИЛ в двулучевой $M_{X}$-схеме КМОН без применения дополнительной фильтрации поляризационных шумов в канале детектирования потенциально позволяет получить предельную вариационную чувствительность на уровне $\sim 11 \mathrm{fT} / \sqrt{\mathrm{Hz}}$ (при выходной оптической мощности $0.8 \mathrm{~mW}$ ). Разработанные ВК-ВИЛ потенциально пригодны для создания компактных цезиевых магнитометрических сенсоров для применения в МЭГ-системах.

\section{Конфликт интересов}

Авторы заявляют, что у них нет конфликта интересов.

\section{Список литературы}

[1] M. Hämäläinen, R. Hari, R.J. Ilmoniemi, J. Knuutila, O.V. Lounasmaa, Rev. Mod. Phys., 65 (2), 413 (1993). DOI: 10.1103/RevModPhys.65.413

[2] Magnetoencephalography, ed. by S. Supek, C. Aine (Springer, Cham, 2019). DOI: 10.1007/978-3-030-00087-5_1

[3] I.M. Savukov, M.V. Romalis, Phys. Rev. Lett., 94 (12), 123001 (2005). DOI: 10.1103/PhysRevLett.94.123001

[4] E. Boto, N. Holmes, J. Leggett, G. Roberts, V. Shah, S.S. Meyer, L.D. Muñoz, K.J. Mullinger, T.M. Tierney, S. Bestmann, G.R. Barnes, R. Bowtell, M.J. Brookes, Nature, 555 (7698), 657 (2018). DOI: 10.1038/nature26147

[5] A. Ossadtchi, N. Kulachenkov, D. Chuchelov, A. Pazgalev, M. Petrenko, A. Vershovskii, in Proc. of Int. Conf. Laser Optics (IEEE, N.Y., 2018), p. 543.

DOI: 10.1109/LO.2018.8435740

[6] W.E. Bell, A.L. Bloom, Phys. Rev., 107 (6), 1559 (1957). DOI: 10.1103/PhysRev.107.1559

[7] N.D. Bhaskar, J. Camparo, W. Happer, A. Sharma, Phys. Rev. A, 23 (6), 3048 (1981). DOI: 10.1103/PhysRevA.23.3048

[8] J. Kitching, Appl. Phys. Rev., 5 (3), 031302 (2018). DOI: $10.1063 / 1.5026238$

[9] F. Gruet, A. Al-Samaneh, E. Kroemer, L. Bimboes, D. Miletic, C. Affolderbach, D. Wahl, R. Boudot, G. Mileti, R. Michalzik, Opt. Express, 21 (5), 5781 (2013).

DOI: $10.1364 /$ OE.21.005781 
[10] VCSELs for cesium-based miniaturized atomic clocks, ed. by A. Al-Samaneh (Books on Demand, 2015). DOI: $10.18725 /$ OPARU-3205

[11] С.А. Блохин, Н.А. Малеев, М.А. Бобров, А.Г. Кузьменков, А.П. Васильев, Ю.М. Задиранов, М.М. Кулагина, А.А. Блохин, Ю.А. Гусева, А.М. Оспенников, М.В. Петренко, А.Г. Гладышев, А.Ю. Егоров, И.И. Новиков, Л.Я. Карачинский, Д.В. Денисов, В.М. Устинов, Квантовая электроника, 49 (2), 187 (2019). [S.A. Blokhin, N.A. Maleev, M.A. Bobrov, A.G. Kuz'menkov, A.P. Vasil'ev, Yu.M. Zadiranov, M.M. Kulagina, A.A. Blokhin, Yu.A. Guseva, A.M. Ospennikov, M.V. Petrenko, A.G. Gladyshev, A.Yu. Egorov, I.I. Novikov, L.Ya. Karachinsky, D.V. Denisov, V.M. Ustinov, Quantum Electron., 49 (2), 187 (2019). DOI: 10.1070/QEL16871].

[12] M.A. Bobrov, N.A. Maleev, S.A. Blokhin, A.G. Kuzmenkov, A.P. Vasil'ev, A.A. Blokhin, M.M. Kulagina, Yu.A. Guseva, S.I. Troshkov, V.M. Ustinov, J. Phys.: Conf. Ser., 741, 012078 (2016). DOI: 10.1088/1742-6596/741/1/012078

[13] С.А. Блохин, М.А. Бобров, А.Г. Кузьменков, А.А. Блохин, А.П. Васильев, Ю.А. Гусева, М.М. Кулагина, Ю.М. Задиранов, Н.А. Малеев, И.И. Новиков, Л.Я. Карачинский, Н.Н. Леденцов, В.М. Устинов, Письма в ЖТФ, 44 (1), 67 (2018). DOI: 10.21883/PJTF.2018.01.45432.17029 [S.A. Blokhin, M.A. Bobrov, A.G. Kuz'menkov, A.A. Blokhin, A.P. Vasil'ev, Yu.A. Guseva, M.M. Kulagina, Yu.M. Zadiranov, N.A. Maleev, I.I. Novikov, L.Ya. Karachinsky, N.N. Ledentsov, V.M. Ustinov, Tech. Phys. Lett., 44 (1), 28 (2018). DOI: $10.1134 / \mathrm{S} 1063785018010042]$.

[14] E. Kroemer, J. Rutkowski, V. Maurice, R. Vicarini, M.A. Hafiz, C. Gorecki, R. Boudot, Appl. Opt., 55 (31), 8839 (2016). DOI: 10.1364/AO.55.008839

[15] D. Budker, M. Romalis, Nature Phys., 3 (4), 227 (2007). DOI: $10.1038 /$ nphys 566

[16] M.A. Bobrov, S.A. Blokhin, N.A. Maleev, A.A. Blokhin, A.P. Vasil'ev, A.G. Kuzmenkov, A.S. Pazgalev, M.V. Petrenko, S.P. Dmitriev, A.K. Vershovskii, V.M. Ustinov, I.I. Novikov, L.Ya. Karachinskii, J. Phys.: Conf. Ser., 1697, 012175 (2020). DOI: 10.1088/1742-6596/1697/1/012175

[17] R. Zhang, R. Mhaskar, K. Smith, M. Prouty, Appl. Phys. Lett., 116 (14), 143501 (2020). DOI: 10.1063/5.0004746 